\title{
X609, a novel manassantin A derivative, exhibits antitumor activity in MG-63 human osteosarcoma cells in vitro and in vivo
}

\author{
$\mathrm{JI} \mathrm{LI}^{1,2^{*}}, \mathrm{KONGBO} \mathrm{ZHAO}^{3 *}, \mathrm{FU} \mathrm{WANG}^{4}, \mathrm{JINFANG} \mathrm{CAI}^{2}$, ZONGYU $\mathrm{LI}^{2}$ and $\mathrm{LIN} \mathrm{ZOU}^{2}$ \\ ${ }^{1}$ Department of Orthopedics, The Quanzhou Licheng Xingxian Hospital, Quanzhou 362005; ${ }^{2}$ Department of Orthopedics, \\ The Jinan Military General Hospital, Jinan 250031; ${ }^{3}$ Department of Orthopedics, Linyi People's Hospital, Linyi 276000; \\ ${ }^{4}$ Department of Orthopedics, Shandong Provincial Hospital, Jinan 250021, P.R. China
}

Received July 26, 2014; Accepted April 10, 2015

DOI: $10.3892 / \mathrm{mmr} .2015 .3729$

\begin{abstract}
Manassantin A has been well-established as an inhibitor of HIF-1. In the present study, a new manasantin A derivative, X609, with decreased stereochemical complexity, rendering it amenable to a simplified synthesis scheme, was synthesized and was found to increase HIF-1 inhibitory activity. X609 exhibited antiproliferative activity in a broad spectrum of tumor cell lines, via HIF-1-dependent mechanisms. X609 may induce apoptosis in MG-63 cells through activation of the mitochondrial pathway. Oral administration of X609 significantly inhibited the growth of human osteosarcomas implanted into nude mice. In light of the results of the present study, it may be possible to develop X609 for use as a novel antitumor agent, which targets human osteosarcoma.
\end{abstract}

\section{Introduction}

Osteosarcoma (OS), which has a poor prognosis in children and adolescents, is the most common primary malignant bone tumor, and predominantly occurs in the metaphyses of long bones (1). Although the application of adjuvant chemotherapy with cisplatin, doxorubicin and methotrexate in the treatment of OS has significantly improved the survival of patients with this disease, there are difficulties with the use of this treatment, including severe side effects and the development of chemoresistance $(2,3)$. The overall 5 -year survival rate is $\sim 65 \%$, while the rates following recurrence or metastasis are $<35 \%$ (4).

Correspondence to: Dr Fu Wang, Department of Orthopedics, Shandong Provincial Hospital, 324 Jing 5 Wei 7 Road, Jinan 250021, P.R. China

E-mail: fuwangjn@163.com

Dr Jinfang Cai, Department of Orthopedics, The Jinan Military General Hospital, 50 Shifan Road, Jinan 250031, P.R. China

E-mail: jinfangcaijf@163.com

*Contributed equally

Key words: manassantin A derivative, hypoxia inducible factor-1 inhibitor, human osteosarcoma, MG-63 cell line, nude mice
Thus, agents with novel mechanisms of action should be developed, in order to establish safer and more effective therapeutic strategies for osteosarcoma.

It has been shown that oxygen is unable to effectively diffuse throughout a solid tumor when the tumor volume exceeds a critical value of $1 \mathrm{~mm}^{3}$. In order to sustain rapid growth, adaptation to hypoxia by tumor cells is particularly important (5). Hypoxia-inducible factor (HIF), the primary regulator of the cellular hypoxia response, is involved in this process (6). A number of HIF-1 target genes are known to promote cell survival under hypoxic conditions, and HIF is overexpressed in various types of cancer (7). Therefore, inhibition of HIF activity represents an viable strategy for the treatment of cancer. To date, a number of HIF inhibitors have been developed and used in clinical trials (8).

Manassantin A, a dineolignan isolated from Saururus cernuиs (Saururaceae), has been shown to inhibit NF- $\mathrm{KB}$ activation and nitric oxide production in macrophages, and to block MAPK activation in mast cells $(9,10)$. Recently, manassantin A was identified as an HIF inhibitor, with $\mathrm{IC}_{50}$ values ranging from 1-10 nM, which inhibits HIF activity by blocking HIF accumulation without altering HIF transcription (11). Notably, manassantin A exhibited antiproliferative activities against cancer cell lines, with $\mathrm{IC}_{50}$ values from 1-1,000 nM, indicating the potential of this compound for further development as an antineoplastic agent (12).

In the current study, a compound termed X609 was developed, the activities of this novel manassantin A derivative were compared with manassantin $\mathrm{A}$, and its anti-tumor mechanisms were investigated. The current study aimed to provide novel insight into the potential development of X609 as a therapeutic agent for osteosarcoma treatment.

\section{Materials and methods}

Materials. The cell lines used (presented in Table I) in this study were obtained from the American Type Culture Collection (Manassas, VA, USA) or Shanghai Cell Bank (Shanghai, China). DMEM, F12, RPMI-1640 and fetal bovine serum (FBS) were purchased from Gibco BRL (Gaithersburg, MD, USA). 3-(4,5-dimethylthiazol)-2,5-diphenyltetrazolium-bromide (MTT) was obtained from Sigma-Aldrich (St. Louis, MO, USA). 5,5',6,6'-tetrachloro-1,1',3,3'-tetraethylbenzimidazolylcar- 
bocyanine iodide (JC-1) was obtained from Molecular Probes Life Technologies (Carlsbad, CA, USA). The cytochrome $c$ enzyme-linked immunosorbentassay kit was obtained from R\&D systems (Minneapolis, MN, USA). Caspase-3, caspase- 8 and caspase-9 activity assay kits were obtained from Kaiji Institute of Biological Engineering (Nanjing, China). The Cell Death Detection ELISA plus kit was from obtained Roche Applied Sciences (Basel, Switzerland). Primary monoclonal antibodies (rabbit anti-caspase-3, \#9665, 1:2,000; rabbit anti-caspase-8, \#4790, 1:2,000; mouse anti-caspase-9, \#9508, 1:2,000; rabbit anti-Bcl-2, \#2870, 1:3,000; and rabbit anti-Bax, \#5023, 1:3,000) were purchased from Cell Signaling Technology, Inc. (Danvers, MA, USA) and secondary goat anti-rabbit (NEF812001EA, 1:5,000) and goat anti-mouse (NEF822001EA, 1:5,000) horseradish peroxidase-labeled $\mathrm{IgG}$ antibodies were purchased from PerkinElmer, Inc. (Boston, MA, USA). Taxol and cisplatin were obtained from Lukang Pharmaceutical Co., Ltd. (Jining, China). All solvents and other chemicals used in this study were of analytical grade and were obtained from Sinopharm chemical reagent Co., Ltd. (Shanghai, China). X609 was synthesized and identified by the institute of Materia Medica, Shangdong Academy of Medical Sciences (Jinan, China), and had a purity of $98 \%$. For the in vitro experiments, treated chemicals were dissolved in DMSO (Sigma-Aldrich). For the animal experiments, taxol and cisplatin were prepared freshly using sterile saline. X609 was prepared in $0.5 \%$ carboxymethylated cellulose as a stock solution, and stored was at $-20^{\circ} \mathrm{C}$ prior to use.

HIF-1 reporter activity and vascular endothelial growth factor (VEGF) protein expression assays. T47D cells were cotransfected with $0.01 \mu \mathrm{g}$ pRL-CMV (Promega Corporation, Madison, WI, USA) and $0.2 \mu \mathrm{g}$ pGL2-TK-HRE, with a firefly luciferase gene driven by 3 tandem repeats of HRE sequences (13), using Lipofectamine 2000 (Invitrogen Life Technologies, Carlsbad, CA, USA) according to the manufacturer's instructions. After $24 \mathrm{~h}$, cells were treated by test compounds for $30 \mathrm{~min}$, moved to a hypoxia chamber $\left(5 \% \mathrm{CO}_{2}, 1 \% \mathrm{O}_{2}, 94 \% \mathrm{~N}_{2}\right)$, and incubated for $16 \mathrm{~h}$ for the dual luciferase activity assays. VEGF protein expression was measured using an ELISA assay. T47D cells were treated as above, and conditioned media at $37^{\circ} \mathrm{C}$ were transferred to a new plate for $16 \mathrm{~h}$. The quantity of VEGF was determined according to the manufacturer's instructions (R\&D Systems).

Cell culture and viability assay. Cells were maintained in DMEM, DMEM/F12 or RPMI-1640 medium, supplemented with $10 \%$ FBS, $100 \mathrm{U} / \mathrm{mL}$ Penicillin and $100 \mu \mathrm{g} / \mathrm{mL}$ Streptomycin, at $37^{\circ} \mathrm{C}$ in humidified $95 \%$ air $/ 5 \% \mathrm{CO}_{2}$. Cell viability following treatments with tested compounds (manassantin A, $10 \mathrm{nM}-10 \mu \mathrm{M}$; X609, $10 \mathrm{nM}-10 \mu \mathrm{M}$; taxol, 1-100 nM) for $72 \mathrm{~h}$ were determined using an MTT assay, as described previously (14). Briefly, cells in the 96-well plate were incubated with $0.5 \mathrm{mg} / \mathrm{ml} \mathrm{MTT} \mathrm{solution} \mathrm{for} 4 \mathrm{~h}$. MTT was then dissolved in DMSO. The absorbance was measured at $570 \mathrm{~nm}$, using a microplate reader (BioTek Instruments, Inc., Winooski, VT, USA). IC $_{50}$ values were calculated using the software GraphPad Prism 5.0.

Measurement of mitochondrial membrane potential (MMP). The MMP was measured using the fluorescent probe, JC-1.
The ratio between aggregate (red) and monomer (green) fluorescence reflects the membrane potential of the mitochondria. Following treatment, MG-63 cells were washed twice with PBS and incubated with JC-1 for $15 \mathrm{~min}$ at $37^{\circ} \mathrm{C}$ in darkness. After rinsing twice, the red/green fluorescence intensity of the cells was determined using a fluorescence microplate reader (Tecan Polarion, Tecan, Reading, UK), at an excitation of $490 \mathrm{~nm}$, and an emission of $530 \mathrm{~nm}$ (green fluorescent monomers) or $590 \mathrm{~nm}$ (red fluorescent aggregates). The change in MMP was expressed as a percentage of the negative control, which was set to $100 \%$.

Cytochrome c assay. Cytosolic cytochrome $c$ was measured using the assay kit. Following treatment, MG-63 cells were washed twice with PBS and fractionated. Cytochrome $c$ was measured according to the manufacturer's instructions. The optical density was measured using a microplate reader (3550; Bio-Rad Laboratories, Inc., Hercules, CA, USA) at $490 \mathrm{~nm}$. The level of cytochrome $c$ was expressed as a percentage of the negative control, which was set to $100 \%$.

Measurement of DNA fragmentation. Quantification of DNA fragmentation was measured using the Cell Death Detection ELISA plus kit. Following treatment, MG-63 cells were washed twice and lysed for $30 \mathrm{~min}$. Cell extracts were centrifuged at $50 \mathrm{x} \mathrm{g}$ for $10 \mathrm{~min}$ at $4^{\circ} \mathrm{C}$. The supernatant $(20 \mu \mathrm{l})$ was transferred to a streptavidin-coated microplate, and incubated with a mixture of anti-histone-biotin and anti-DNA-peroxidase (Roche Diagnostics Corporation, Indianapolis, IN, USA). The peroxidase in the immunocomplex was quantified by adding 2,2'-azinobis (3-ethylbenzthiazoline-6-sulfonic acid) (Sigma-Aldrich) as the substrate. The absorbance was measured using a microplate reader at $405 \mathrm{~nm}$. The extent of DNA fragmentation was expressed as a percentage of the negative control, which was set to $100 \%$.

Measurement of caspase activity. Caspase activity was measured using the assay kit, according to the manufacturer's instructions. Following treatment, MG-63 cells were washed twice with PBS and lysed with a buffer, consisting of $1 \mathrm{mM}$ EGTA-Na, $2 \mathrm{mM} \mathrm{MgCl}$, 25 mM HEPES (pH 7.5), 0.1\% CHAPS, 2 mM DTT, 100 mM PMSF and 1:100 broad spectrum protease inhibitor. Following centrifugation at $45,000 \mathrm{x} \mathrm{g}$ for $10 \mathrm{~min}$ at $4^{\circ} \mathrm{C}$, aliquots of supernatants containing $25 \mathrm{mg}$ protein were added to a reaction buffer that was supplemented with $0.1 \%$ CHAPS, $100 \mathrm{mM}$ PMSF and $5 \mathrm{mM}$ DTT. The reactions were initiated following addition of the following fluorescent substrates $(50 \mathrm{mM}$ final concentration): Ac-DEVD-Amc, for caspase-3 activity; Ac-IETD-Amc, for caspase-8 activity; and Ac-LEDH-Afc, for caspase- 9 activity. After incubating for $2 \mathrm{~h}$ at $37^{\circ} \mathrm{C}$, the cleavage of the substrates was measured (Amc: 390/475 nm; Afc: $400 / 505 \mathrm{~nm}$ ) using a microplate reader. The activity of caspase was expressed as a percentage of the negative control, which was set to $100 \%$.

Western blot analysis. Following treatment, MG-63 cells were harvested and washed twice with PBS. Total cellular protein was isolated using the protein extraction buffer and quantified using the protein assay kit (Beyotime Institute of Biotechnology, 
Haimen, China). Cell lysates in 5X SDS sample buffer were boiled for $5 \mathrm{~min}$, and equal amounts of protein $(50 \mu \mathrm{g})$ from each sample were then subjected to electrophoresis on a $12 \%$ (v/v) SDS-polyacrylamide gel (Bio-Rad Laboratories, Inc.). Following electrophoresis, proteins were electroblotted onto a PVDF membrane (EMD Millipore, Temecula, CA, USA). After blocking with PBST (Sigma-Aldrich) containing $5 \%$ dried non-fat milk at room temperature, membranes were washed three times and incubated with the indicated primary antibodies at $4^{\circ} \mathrm{C}$ overnight, followed by incubation with the appropriate horseradish peroxidase-conjugated secondary antibody for $1 \mathrm{~h}$. Following incubation, membranes were washed three times, and the antigen-antibody complexes were visualized using the enhanced chemiluminescence system (PerkinElmer). The relative protein expression, with the control defined as $100 \%$, was measured using ImageJ software (National Institutes of Health, Bethseda, MA, USA).

Xenograft tumor growth. The animal experiment was approved by the Ethics Committee of The Jinan Military General Hospital (Jinan, China). Female nude mice (BALB/c-nu/nu; 7 weeks old) were obtained from the Experimental Animal Center of Shandong (Jinan, China) and were housed in a sterile environment at $25^{\circ} \mathrm{C}$ on a 12-h light/dark cycle. MG-63 cells $\left(1 \times 10^{7}\right)$ were injected subcutaneously into the right flank of five nude mice. After being allowed to grow for 3-4 weeks, nude mice were sacrificed by $\mathrm{CO}_{2}$ inhalation, tumors were detached and weighed, cut into $2 \times 2 \times 2 \mathrm{~mm}^{3}$ pieces, and implanted subcutaneously into the right flank of 40 nude mice with a gauge trocar. When the tumor volume reached to $100-200 \mathrm{~mm}^{3}$, mice were randomized into four groups (8 mice per group: control, cisplatin, X609 $50 \mathrm{mg} / \mathrm{kg}$ and X609 $100 \mathrm{mg} / \mathrm{kg}$ groups), and treated with the tested compounds (X609, $50 \mathrm{mg} / \mathrm{kg}$ and $100 \mathrm{mg} / \mathrm{kg}$; cisplatin, $5 \mathrm{mg} / \mathrm{kg}$ ). Cisplatin $(5 \mathrm{mg} / \mathrm{kg}$ ) was injected twice weekly intraperitoneally for three weeks. X609 was administered orally for 6 days per week for three weeks. Tumor volume was calculated as described previously (15).

Statistical analysis. Data are presented as the mean \pm standard deviation, and were analyzed using one-way analysis of variance, with the Sigma Stat statistical software, version 4.0 (SPSS Inc., Chicago, IL, USA). P $<0.05$ was considered to indicate a statistically significant difference.

\section{Results}

Novel manassantin A derivative, X609, inhibits HIF-1 transcription activity. Manassantin $\mathrm{A}$ is an efficacious HIF-1 inhibitor. Based on its structure, a novel derivative, X609 (Fig. 1A), was designed and synthesized for use in the present study. Its HIF-1 inhibitory activity was determined using a cell-based HIF-1 reporter assay. Compared with the eight chiral centers of manassantin A, X609 has only two chiral centers, meaning it is easier to prepare and purify. Furthermore, this novel derivative inhibited HIF-1 activity more effectively than manassantin $\mathrm{A}\left(\mathrm{IC}_{50}, 0.32 \mathrm{nM}\right.$ and $58 \mathrm{nM}$, respectively). In accordance with this HIF-1 inhibitory activity, X609 significantly downregulated the hypoxia-induced expression of VEGF, a known HIF-1 target gene (Fig. 1B).
A
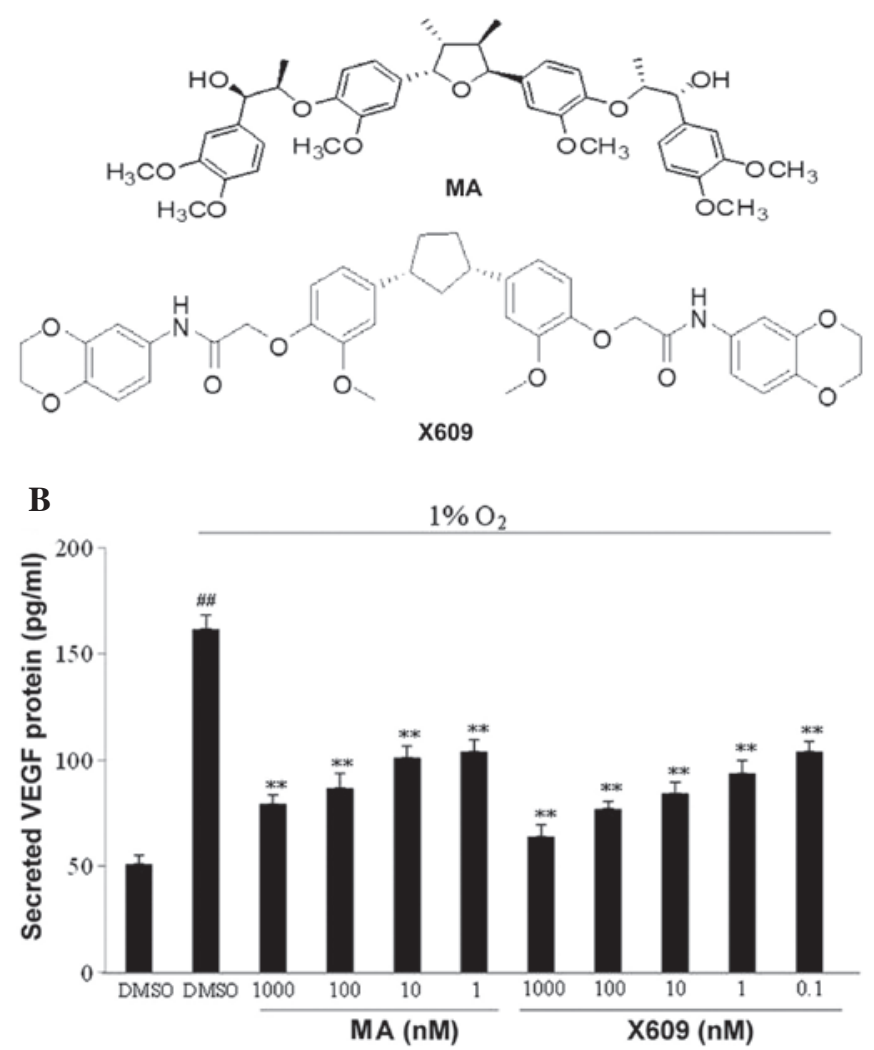

Figure 1. X609 is a novel HIF inhibitor, derived from MA. (A) Structures of MA and X609. (B) X609 inhibited VEGF protein secretion in T47D cells. T47D cells were treated with MA and X609, and then incubated in a hypoxia chamber $\left(1 \% \mathrm{O}_{2}\right)$ for $16 \mathrm{~h}$. The quantity of VEGF was determined using an ELISA assay. Data are presented as the mean \pm standard deviation of three independent experiments. ${ }^{\# \#} \mathrm{P}<0.01$, vs DMSO (normoxic condition group) and ${ }^{* *} \mathrm{P}<0.01$, vs DMSO ( $1 \% \mathrm{O}_{2}$ group). VEGF, vascular endothelial growth factor, MA, manassantin A.

X609 inhibits the growth of a variety of cancer cell lines. In addition to the effect on HIF-1 activity, the antiproliferative activities of X609 on 12 different cancer cell lines was investigated using an MTT assay. Manassantin A and X609 each selectively inhibited the growth of osteosarcoma, and lung and pancreatic cancer cell lines. Notably, X609 appeared to exhibit a more potent antiproliferative activity than manassantin $\mathrm{A}$, as the $\mathrm{IC}_{50}$ values of this compound were generally 2-20-fold less than those of manassantin A (Table I). Furthermore, the $\mathrm{IC}_{50}$ values under hypoxic conditions were lower than those under normoxic conditions, suggesting that X609 may inhibit cell growth through an HIF-1-dependent mechanism.

X609 induces MG-63 cell apoptosis. X609 significantly reduced cell viability, as demonstrated by the MTT assay. In order to explore the mechanisms underlying its antitumor activity, the expression of apoptosis-related markers was measured. Treatment with X609 led to an marked decrease in the MMP (Fig. 2A); significantly increased the quantity of cytochrome $c$ released into the cytoplasm (Fig. 2B); increased the DNA fragmentation in MG-63 cells (Fig. 2C); and enhanced the activities of caspase- 3 , caspase- 8 and caspase-9 (Fig. 2D), compared with the control group. Western 
Table I. Inhibition of growth of cultured cancer cells by X609.

\begin{tabular}{llcccccc}
\hline & & \multicolumn{2}{c}{$\begin{array}{c}\text { Growth inhibition under normoxic } \\
\text { conditions }\left(\mathrm{IC}_{50}, \mathrm{nM}\right)\end{array}$} & & \multicolumn{2}{c}{$\begin{array}{c}\text { Growth inhibition under hypoxic } \\
\text { conditions }\left(\mathrm{IC}_{50}, \mathrm{nM}\right)\end{array}$} \\
Cell Line & \multicolumn{1}{c}{ Origin } & Manassantin A & X609 & Taxol & & Manassantin A & X609 \\
\hline HepG2 & Hepatocellular & $>1000$ & $>1000$ & $25.6 \pm 9.2$ & & $>1000$ & $>1000$ \\
U251 & Glioblastoma & $>1000$ & $>1000$ & $8.7 \pm 6.2$ & & $>1000$ & $>1000$ \\
A375 & Melanoma & $>1000$ & $>1000$ & $25.3 \pm 11.5$ & & $>1000$ & $>1000$ \\
HT-29 & Colon adenocarcinoma & $>1000$ & $>1000$ & $7.3 \pm 2.8$ & & $>1000$ & $>1000$ \\
BGC-823 & Stomach adenocarcinoma & $>1000$ & $>1000$ & $8.3 \pm 3.1$ & & $>1000$ & $>1000$ \\
A549 & Lung adenocarcinoma & $251.2 \pm 68.3$ & $21.5 \pm 7.1$ & $22.6 \pm 8.7$ & & $108.5 \pm 56.3$ & $9.5 \pm 3.4$ \\
NCI-H460 & Lung adenocarcinoma & $895.7 \pm 231.6$ & $361.7 \pm 97.6$ & $76.3 \pm 21.8$ & & $354.5 \pm 152.1$ & $152.8 \pm 52.3$ \\
MCF-7 & Breast adenocarcinoma & $>1000$ & $>1000$ & $4.9 \pm 1.3$ & & $>1000$ & $>1000$ \\
T47D & Breast adenocarcinoma & $754.2 \pm 214.3$ & $97.3 \pm 30.5$ & $6.2 \pm 4.1$ & & $251.6 \pm 117.5$ & $45.8 \pm 14.6$ \\
PANC-1 & Pancreatic adenocarcinoma & $298.7 \pm 132.5$ & $69.5 \pm 15.8$ & $2.7 \pm 1.2$ & & $112.8 \pm 46.3$ & $26.8 \pm 8.3$ \\
MG-63 & Osteosarcoma & $257.9 \pm 125.2$ & $12.7 \pm 3.4$ & $10.3 \pm 2.6$ & & $78.7 \pm 27.5$ & $5.4 \pm 1.8$ \\
HOS & Osteosarcoma & $328.5 \pm 175.8$ & $15.3 \pm 4.9$ & $11.2 \pm 3.7$ & & $132.1 \pm 43.4$ & $6.6 \pm 2.1$ \\
\hline
\end{tabular}

A

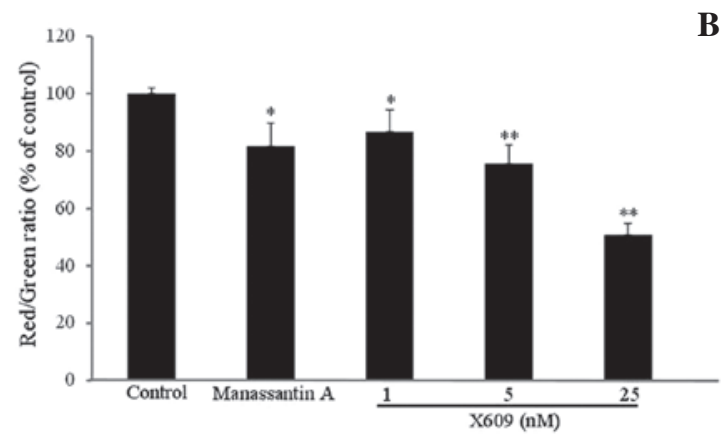

C

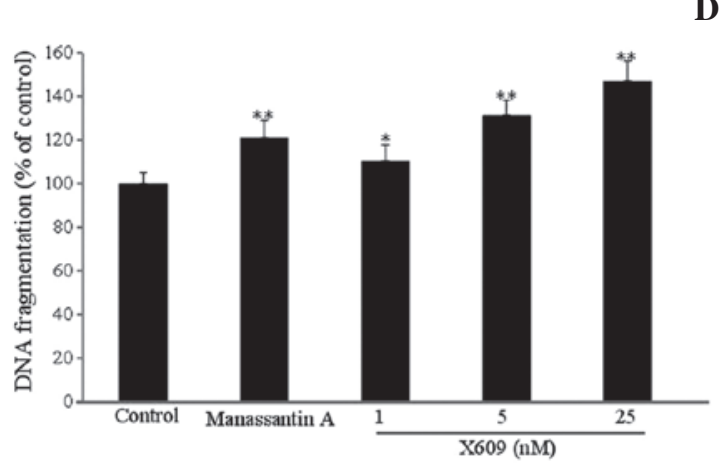

B

D
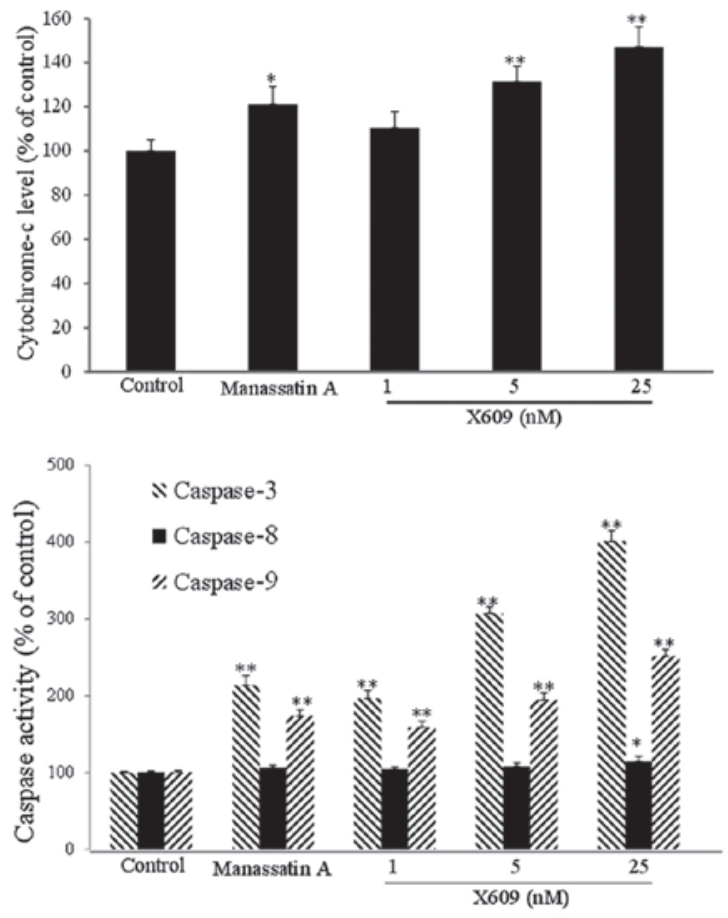

$\mathbf{E}$

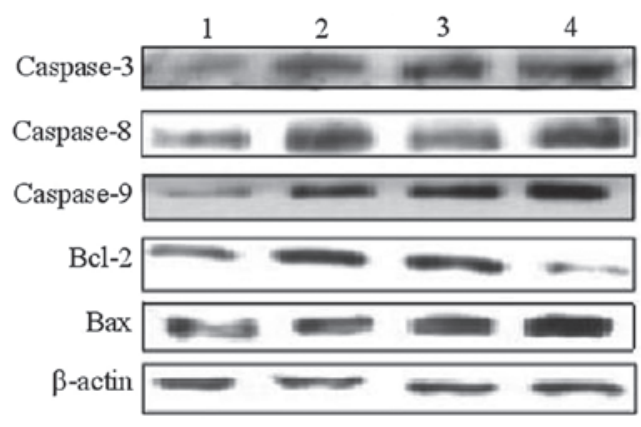

Figure 2. X609 induced apoptosis in MG-63 cells. MG-63 cells were treated with X609 or manassantin A (10 nM) for 48 h. (A) Effect of X609 on MMP in MG-63 cells assayed by JC-1. JC-1 is sensitive to MMP, and the changes in the ratio between aggregate (red) and monomer (green) fluorescence provide information regarding changes in the MMP. (B) Effect of X609 on cytochrome $c$ release in MG-63 cells. (C) Effect of X609 on DNA fragmentation in MG-63 cells. (D) Effect of X609 on caspase activity in MG-63 cells. (E) Effect of X609 on the expression of apoptosis-related proteins. Data are expressed as the mean \pm standard deviation of three independent experiments. ${ }^{*} \mathrm{P}<0.05$ and ${ }^{* *} \mathrm{P}<0.01$, vs the control group. MMP, mitochondrial membrane potential. 
A

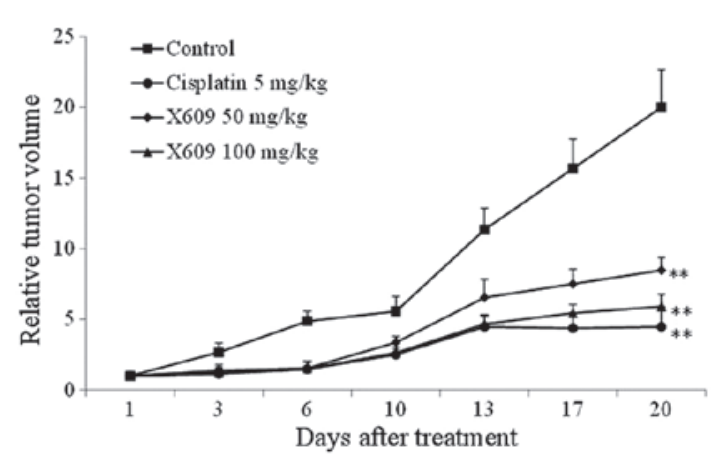

C
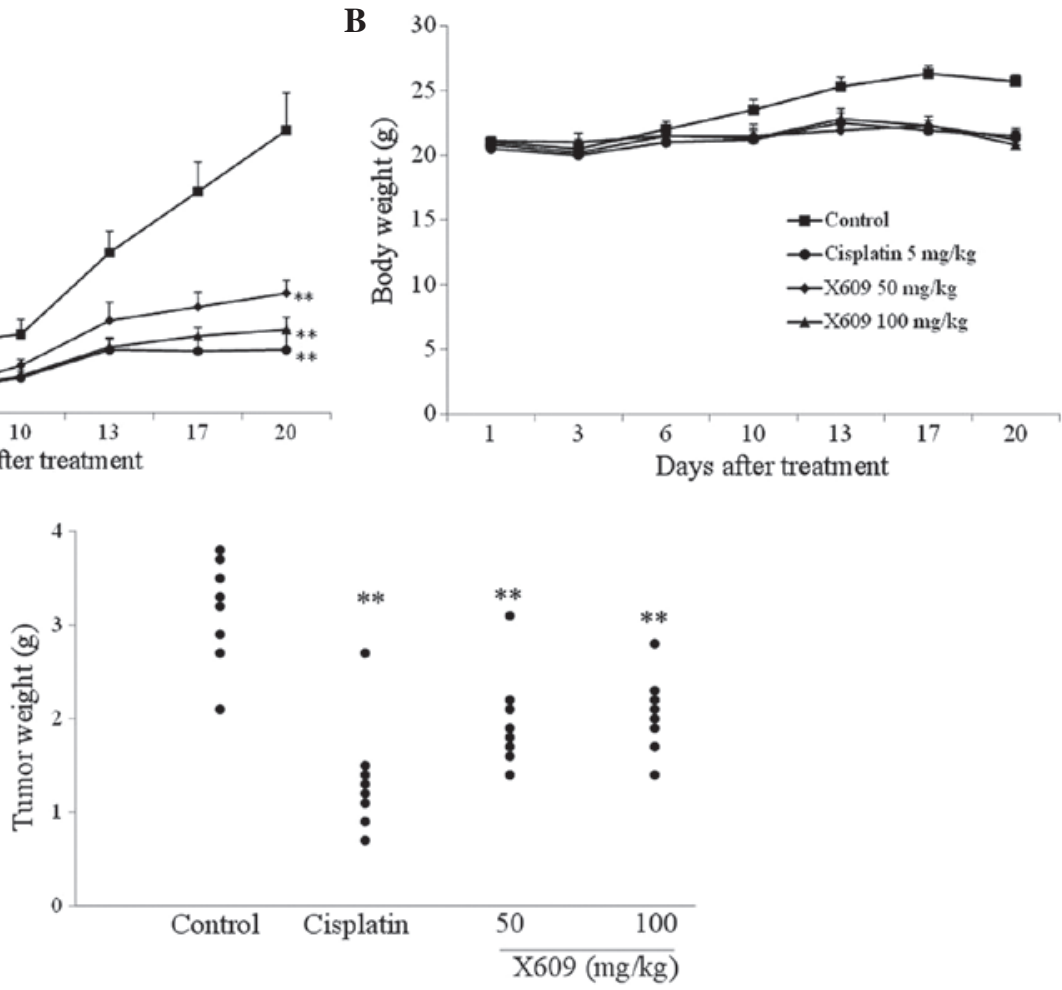

Figure 3. X609 inhibited human osteosarcoma xenograft tumor growth. Nude mice with MG-63 xenografts were treated with X609 or cisplatin. (A) Tumor volume was measured and used to calculate the relative tumor volume. (B) Body weights were monitored. (C) Nude mice were sacrificed at the end of the experiment, and tumors were dissected out and weighed. ${ }^{* *} \mathrm{P}<0.01$, vs the control group.

blotting results confirmed that the expression of the proapoptotic proteins, caspases and bax, was increased, while that of the antiapopotic protein, bcl-2, was decreased, following treatment with X609 (Fig. 2E). Therefore, X609 appeared to exert its antitumor effect by inducing apoptosis.

X609 inhibits xenograft tumor growth in nude mice. In order to measure the antitumor activity of X609 in vivo, human osteosarcoma MG-63 cells were implanted into nude mice, and mice carrying established tumors were then treated with 50 or $100 \mathrm{mg} / \mathrm{kg}$ of X609. Cisplatin was used as a positive control. At a dose of $100 \mathrm{mg} / \mathrm{kg}$, X609 significantly inhibited growth of MG-63 xenograft tumors (Fig. 3), in terms of tumor volume as well as tumor weight, at the time of mouse sacrifice. The relative tumor volume was reduced to $29.5 \%$ of that of the control, and the tumor weight was reduced to $65 \%$ of that of the control. At a dose of $50 \mathrm{mg} / \mathrm{kg}$, X609 also reduced tumor volume and weight. Notably, X609 had no significant effect on mouse body weight during the experiments, suggesting that X609 is minimally toxic in mice. These results indicate that X609 has the potential for use in the treatment of osteosarcoma.

\section{Discussion}

Osteosarcomas are derived from the mesoderm. It has been hypothesized that osteosarcoma originates from multipotent cells that are able to differentiate into osteoblasts (16). The current strategy for the treatment of high-grade osteosarcoma is based on the administration of neoadjuvant chemotherapy. The initial therapeutic response to the first line of chemotherapy affects the response to subsequent therapeutic agents (17). Thus, there is a requirement to develop chemotherapeutic drugs with novel mechanisms of action.

Manassantin A, an active component in the medicinal herb, Saururaceae, has been identified as a potent HIF-1 inhibitor with various biological activities, including neuroleptic, anti-inflammatory and antitumor effects $(18,19)$. The present study demonstrated that a novel manassantin A derivative, X609, exerted a more potent HIF-1 inhibitory activity than that of manassantin A itself. Furthermore, X609 inhibited the growth of cultured cancer cells, as well as xenograft tumor growth in nude mice. These results suggested that X609 may be suitable for further development as a novel class of anticancer agents, which target HIF-1-mediated prosurvival pathways.

Mitochondria are known to be involved in the regulation of cell death. A number of proteins and genes influence or determine the progression of apoptosis along the mitochondrial pathway (20-22). A change in the mitochondrial membrane potential may induce the release of cytochrome $c$ from the mitochondria into the nucleus, which activates caspase-related proapoptotic proteins and facilitates the formation of an apoptosome complex, leading to chromatin condensation and DNA cleavage. DNA fragmentation is a biomarker of apoptosis and is widely used as an apoptotic index $(23,24)$. Therefore, depolarization of the MMP and the increasing release of cytochrome $c$ from mitochondria were detected, indicating the onset of apoptosis (25). The present study showed that treatment with X609 led to MG-63 cell injury and DNA fragmentation, accompanied by MMP depolarization and cytochrome $c$ release from the mitochondria. 
Caspases are involved in the mitochondrial apoptotic pathway. A variety of stimuli trigger the mitochondrial permeability transition and the release of cytochrome $c$, and the subsequent activation of caspases $(26,27)$. Caspase- 3 acts as an apoptotic executor and activates DNA fragmentation factor, which, in turn, activates endonucleases to cleave nuclear DNA, ultimately resulting in cell death. In addition, caspase-9 is activated following the disruption of the outer mitochondrial membrane, which results in the collapse of the MMP and a consequent change in permeability (28). The present results indicated that exposure of MG-63 cells to X609 significantly increases the activity of caspase- 3 and caspase- 9 . The expression of the proapoptotic proteins, caspases and bax, was increased, while that of the antiapoptotic protein, bcl-2, was decreased, following treatment with X609. These results indicated that X609 may exert its antitumor effect by inducing apoptosis.

In contrast to the results of a previous study (19), in which a manassantin A derivative LXY6006 was able to induce T47D cell cycle arrest but no apoptosis, X609 was observed to induce apoptosis in MG-63 cells via the mitochondrial pathway. The difference may be due to the different side chains between these manassantin A derivatives. In addition, X609 was established to exert more potent anti-proliferative effects under hypoxia, which further indicated an inhibition of cell growth through an HIF-dependent mechanism. The association between the inhibition of HIF and the induction of apoptosis requires further investigation.

X609 was also found to significantly inhibit the growth of human osteosarcoma in nude mice. To the best of our knowledge, these results provide the first evidence supporting the hypothesis that manassantin A derivatives may be used to treat human osteosarcoma in vivo.

\section{Acknowledgements}

This study was supported by the National Natural Science Fund (grant no. 30973018/c1607).

\section{References}

1. Bacci G, Longhi A, Fagioli F, et al: Adjuvant and neoadjuvant chemotherapy for osteosarcoma of the extremities: 27 year experience at Rizzoli Institute, Italy. Eur J Cancer 41: 2836-2845, 2005.

2. Chou AJ, Geller DS and Gorlick R: Therapy for osteosarcoma: Where do we go from here? Pediatric Drugs 10: 315-327, 2008.

3. Bacci G, Longhi A, Versari M, et al: Prognostic factors for osteosarcoma of the extremity treated with neoadjuvant chemotherapy: 15-year experience in 789 patients treated at a single institute. Cancer 106: 1154-1161, 2006.

4. Ottaviani G and Jaffe N: The epidemiology of osteosarcoma. Cancer Treat Res 152: 3-13, 2009.

5. Dang CV and Semenza GL: Oncogenic alterations of metabolism. Trends Biochem Sci 24: 68-72, 1999.

6. Rohwer N, Zasada C, Kempa S and Cramer T: The growing complexity of HIF-1 $\alpha$ 's role in tumorigenesis: DNA repair and beyond. Oncogene 32: 3569-3576, 2013.
7. Blancher $\mathrm{C}$ and Harris AL: The molecular basis of the hypoxia response pathway: Tumour hypoxia as a therapy target. Cancer Metastasis Rev 17: 187-194, 1998.

8. Wilson WR and Hay MP: Targeting hypoxia in cancer therapy. Nat Rev Cancer 11: 393-410, 2011

9. Kim JY, Kang JS, Kim HM, et al: Inhibition of phenotypic and functional maturation of dendritic cells by manassantin a. J Pharmacol Sci 109: 583-592, 2009.

10. Kim SJ, Lu Y, Kwon O, et al: Manassantin A isolated from Saururus chinensis inhibits 5-lipoxygenase-dependent leukotriene $\mathrm{C} 4$ generation by blocking mitogen-activated protein kinase activation in mast cells. Biol Pharm Bull 34: 1769-1772, 2011.

11. Kasper AC, Moon EJ, Hu X, et al: Analysis of HIF-1 inhibition by manassantin $\mathrm{A}$ and analogues with modified tetrahydrofuran configurations. Bioorg Med Chem Lett 19: 3783-3786, 2009.

12. Hahm JC, Lee IK, Kang WK, et al: Cytotoxicity of neolignans identified in Saururus chinensis towards human cancer cell lines. Planta Med 71: 464-469, 2005.

13. Rapisarda A, Uranchimeg B, Scudiero DA, et al: Identificantion of small molecule inhibitors of hypoxia-inducible factor 1 transcriptional activation pathway. Cancer Res 62: 4316-4324, 2002.

14. Lu C, Liu D, Jin J, et al: Inhibition of gastric tumor growth by a novel Hsp90 inhibitor. Biochem Pharmacol 85: 1246-1256, 2013.

15. Zhou Q, Li Y, Jin J, et al: Lx2-32c, a novel taxane derivative, exerts anti-resistance activity by initiating intrinsic apoptosis pathway in vitro and inhibits the growth of resistant tumor in vivo. Biol Pharm Bull 35: 2170-2179, 2012.

16. Heymann D and Redini F: Bone sarcomas: Pathogenesis and new therapeutic approaches. IBMS BoneKey 8: 402-414, 2011.

17. Gobin B, Moriceau G, Ory B, et al: Imatinib mesylate exerts anti-proliferative effects on osteosarcoma cells and inhibits the tumour growth in immunocompetent murine models. PLoS One 9: e90795, 2014.

18. Kwon OE, Lee HS, Lee SW, et al: Manassantin A and B isolated from Saururus chinensis inhibt TNF-alpha-induced cell adhesion molecule expression of human umbilical vein endothelial cells. Arch Pharm Res 28: 55-60, 2005.

19. Lang L, Liu X, Li Y, et al: A synthetic manassantin A derivative inhibits hypoxia-inducible factor 1 and tumor growth. PloS One 9: e99584, 2014.

20. Zhou J and Tang XC: Huperzine A attenuates apoptosis and mitochondria-dependent caspase-3 in rat cortical neurons. FEBS Lett 526: 21-25, 2002.

21. Wang Z, Lu W, Li Y and Tang B: Alpinetin promotes Bax translocation, induces apoptosis through the mitochondrial pathway and arrests human gastric cancer cells at the G2/M phase. Mol Med Rep 7: 915-920, 2013.

22. Lee CS,Han JH, Jang YY, et al: Differential effect of catecholamines and $\mathrm{MPP}(+)$ on membrane permeability in brain mitochondria and cell viability in PC12 cells. Neurochem Int 40: 361-369, 2002.

23. Yuan J, Adamski R and Chen J: Focus on histone variant H2AX: To be or not to be. FEBS Lett 584: 3717-3724, 2010.

24. Wang $\mathrm{C}$ and Youle RJ: The role of mitochondria in apoptosis. Annu Rev Genet 43: 95-118, 2009.

25. Gannavaram S and Debrabant A: Programmed cell death in Leishmania: Biochemical evidence and role in parasite infectivity. Front Cell Infect Microbiol 2: 95, 2012.

26. Liu XY, Xu KD, Yan M, et al: Protective effects of galantamine against Abeta-induced PC12 cell apoptosis by preventing mitochondrial dysfunction and endoplasmic reticulum stress. Neurochem Int 57: 588-599, 2010.

27. Kabeer FA, Sreedevi GB, Nair MS, et al: Isodeoxyelephantopin from Elephantopus scaber (Didancao) induces cell cycle arrest and caspase-3-mediated apoptosis in breast carcinoma T47D cells and lung carcinoma A549 cells. Chin Med 9: 14, 2014.

28. Stratford EW, Daffinrud J, Munthe E, et al: The tankyrase-specific inhibitor JW74 affects cell cycle progression and induces apoptosis and differentiation in osteosarcoma cell lines. Cancer Med 3: 36-46, 2014. 